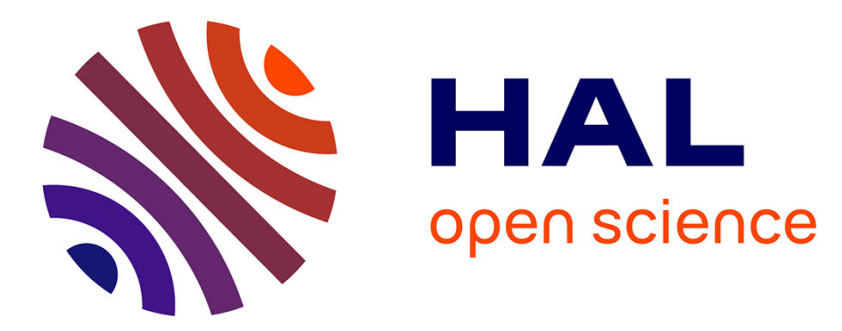

\title{
PROGRAMMATION MOTRICE ET STRATÉGIES COGNITIVES DANS UNE TÂCHE DE SYNCHRONISATION
}

Catherine Auxiette, Corinne Gerard

\section{- To cite this version:}

Catherine Auxiette, Corinne Gerard. PROGRAMMATION MOTRICE ET STRATÉGIES COGNITIVES DANS UNE TÂCHE DE SYNCHRONISATION. Journal de Physique IV Proceedings, 1992, 02 (C1), pp.C1-225-C1-228. 10.1051/jp4:1992146 . jpa-00251217

\section{HAL Id: jpa-00251217 https://hal.science/jpa-00251217}

Submitted on 1 Jan 1992

HAL is a multi-disciplinary open access archive for the deposit and dissemination of scientific research documents, whether they are published or not. The documents may come from teaching and research institutions in France or abroad, or from public or private research centers.
L'archive ouverte pluridisciplinaire HAL, est destinée au dépôt et à la diffusion de documents scientifiques de niveau recherche, publiés ou non, émanant des établissements d'enseignement et de recherche français ou étrangers, des laboratoires publics ou privés. 


\title{
PROGRAMMATION MOTRICE ET STRATÉGIES COGNITIVES DANS UNE TÂCHE DE SYNCHRONISATION
}

\author{
C. AUXIETTE et C. GERARD \\ Laboratoire de Psychologie Expérimentale, CNRS URA 316, Université René Descartes, 28 rue \\ Serpente, F-75006 Paris, France
}

\begin{abstract}
Résumé - Les anticipations généralement observées dans une tâche de synchronisation ne sont pas toujours systématiques, ni de valeur constante. Tout dépend de la nature des sons (présentés et produits), de la vitesse des séquences et des stratégies des sujets.

Abstract - Anticipation is usually observed in synchronization tasks. However, it is neither systematic nor of constant value, and varies with the nature of the sounds used as well as with the tempo of the sequence and the subjects'. strategies.
\end{abstract}

\section{INTRODUCTION}

De nombreuses expériences de synchronisation dans lesquelles des sujets doivent faire coïncider leurs frappes avec un clic de métronome montrent une anticipation systématique des frappes par rapport aux clics du métronome [1,2,3], et soulignent l'existence de difficultés a) sensorielles dans la perception de la succession pour des vitesses rapides, b) motrices dans la production de cadences élevées [4,5], et c) mnésiques, particulièrement pour la mémorisation des intervalles longs [4]. La synchronisation optimale s'observe pour des intervalles inter-stimulus (IIS) de 400 à $800 \mathrm{~ms}$ [1]: 1'écart frappes-sons ne dépasse alors pas $50 \mathrm{~ms}$, valeur inférieure au seuil de la discrimination de la succession qui est d'environ $60 \mathrm{~ms}$ [5,6]. Pour des cadences très rapides, les difficultés motrices rendent la performance hasardeuse et les écarts frappes-sons variables. Lorsque les IIS dépassent une seconde, les écarts frappes-sons augmentent considérablement et dépassent de beaucoup le seuil de discrimination de la succession. Nombreuses sont également les expériences où l'on demande à des sujets soit de produire des monosyllabes (comme $/ \mathrm{ba} /$ et $/ \mathrm{ma} /$ ) en alternance, de manière régulière, soit de juger de l'isochronie d'une séquence de monosyllabes. Les résultats des expériences de production sont assez concordants: les productions des sujets ne sont pas acoustiquement isochrones, les intervalles entre les syllabes, mesurés d'onset à onset, n'étant pas équidistants $[7,8,9,10]$. Les résultats des expériences de perception montrent que les auditeurs jugent des séquences de monosyllabes acoustiquement isochrones comme non régulières, et inversement des séquences de syllabes non isochrones acoustiquement sont perçues isochrones $[8,9]$. Ces recherches suggèrent l'existence de "P-Center". c'est-à-dire de points internes à la syllabe qui jouent le rôle de "centre de gravité" de cette unité sonore et dont la localisation varie suivant la composition de la syllabe [11]. Toutes ces études concernent l'adulte.

Notre expérience examine la synchronisation entre des sons parlés et des clics de métronome, les uns et les autres étant tantôt stimulus et tantôt réponse, afin de vérifier que dans une tâche de synchronisation, c'est ce que le sujet produit qui est anticipé sur ce qu'il entend. Cependant, le nombre et la valeur des anticipations doivent dépendre de la nature des sons 
utilisés, des intervalles entre les sons stimulus, de la qualité du contrôle perceptif et moteur que peut réaliser le sujet en fonction de son âge.

\section{METHODE}

Seize enfants de 6 ans et onze adultes devaient produire pendant une minute soit des sons parlés (syllabe ti ou ta) soit des sons frappés (appelés "bip") en même temps qu'une séquence stimulus isochrone composée soit de sons parlés soit de bips. Cinq conditions ont été créées: bip/bip (stimulus et réponse sont des bips), ti/bip et ta/bip (stimulus $=$ syllabe, réponse $=$ bip), bip/ti et bip/ta (stimulus $=$ bip, réponse $=$ syllabe). Deux vitesses ont été choisies: l'une lente (IIS $=600 \mathrm{~ms}$ ) et l'autre rapide (IIS $=450 \mathrm{~ms}$ ).

\section{RESULTATS}

Parmi les réponses "synchronisées" (obtenues en excluant les rares réponses situées dans le tiers central de l'intervalle inter-stimulus), nous avons d'une part calculé le pourcentage de réponses anticipées, retardées et parfaitement synchronisées, et d'autre part mesuré les décalages qui séparent l'onset du stimulus de l'onset de la réponse (attaque du signal pour le bip, barre d'explosion pour la syllabe). Les histogrammes ci-dessous (figure 1) présentent les fréquences relatives de chaque type de réponse.
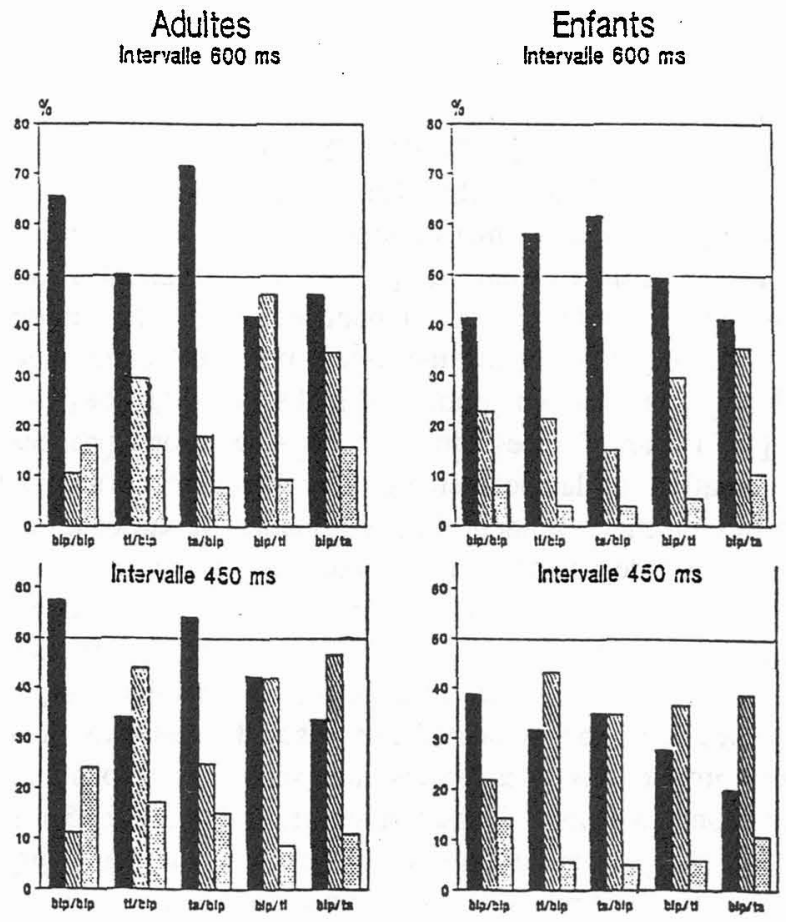

Figure 1 : Pourcentages relatifs de réponses anticipées (E), retardées ( synchronisées ( successivement en abscisse.

A vitesse lente, adultes et enfants anticipent majoritairement leurs réponses, que ces réponses soient frappées ou parlées, mais la fréquence des anticipations est cependant moindre dans les deux conditions bip/ti et bip/ta où la réponse consiste à produire une syllabe. A vitesse 
rapide, cette supériorité manifeste des anticipations ne subsiste que dans la condition bip/bip et les réponses retardées l'emportent dans les autres conditions surtout quand la réponse à produire est une syllabe.

Les histogrammes ci-dessous (figure 2) présentent maintenant les décalages moyens entre stimulus et réponse.
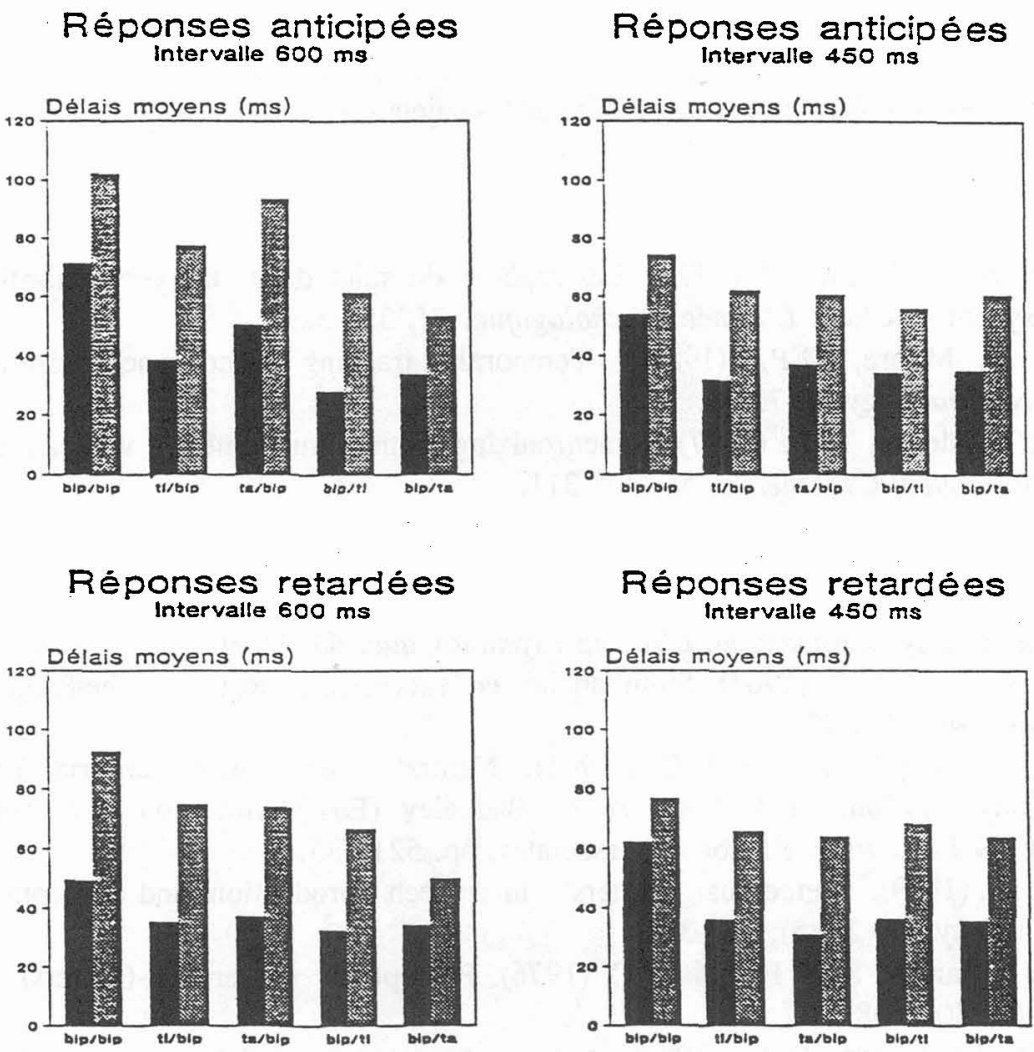

Figure 2 : Délais moyens entre stimulus et réponse des adultes ( $\boldsymbol{D}$ ) et des enfants (榻) pour les 5 conditions bip/bip, ti/bip et ta/bip, bip/ti et bip/ta portées successivement en abscisse.

Les valeurs moyennes des décalages pour les enfants sont toujours supérieures à celles des adultes, témoignant de leur difficulté de contrôle moteur, mais l'organisation des résultats est la même pour les deux groupes de sujets. Dès que la séquence stimulus ou la séquence des réponses contiennent des sons parlés, les décalages temporels - moyens sont toujours plus courts que lorsque la séquence stimulus et la séquence des réponses contiennent seulement des bips (condition bip/bip en première position sur l'abscisse). Aux deux vitesses, le décalage des réponses anticipées aussi bien que retardées est donc lié à des déterminants psychoacoustiques. 


\section{CONCLUSION}

Les fréquences relatives des différentes réponses témoignent de deux types de stratégies : à vitesse dite lente (qui correspond aux marges dans lesquelles évolue le tempo moteur spontané), la gestion des réponses peut s'effectuer au coup par coup et l'anticipation de la main sur la parole se manifeste. A vitesse rapide, où le délai inter-stimulus n'est pas suffisant pour une telle gestion, soit les sujets adoptent une cadence générale conforme à celle des stimuli, soit des retards de la réponse par rapport au stimulus s'observent. La fréquence et l'amplitude de ces anticipations dépendent de la composition acoustique des sons en cause, de la vitesse générale de déroulement de la séquence, des contrôles moteurs réalisés par les sujets.

\section{Références}

[1] Fraisse, P. \& Voillaume, C. (1971). Les repères du sujet dans la synchronisation et dans la pseudo-synchronisation. L'Année Psychologique, 71, 359-369.

[2] Hary, D. \& Moore, G.P. (1985). Temporal tracking and synchronization strategies. Human Neurobiology, 4, 73-79.

[3] Hary, D. \& Moore, G.P. (1987). Synchronizing human movement with an external clock source. Biological Cybernetics, 56, 305-311.

[4] Bartlett, N.R. \& Bartlett, S.C. (1959). Synchronization of a motor response with an anticipated sensory event. Psychological Review, 66 (4), 203-218.

[5] Fraisse, P. (1966). L'anticipation de stimulus rythmiques. Vitesse d'établissement et précision de la synchronisation. L'Année Psychologique, 66, 15-36.

[6] Hirsh, I.J. \& Fraisse, P. (1964). Simultanéité et succession de stimuli hétérogènes. L'Année Psychologique, 64, 1-19.

[7] Fowler, C.A. \& Tassinary, L.G. (1981). Natural measurement criteria for speech : the anisochrony illusion. In J. Long \& A. Baddeley (Eds.), Attention and Performance, IX. Hillsdale, N.J : Lawrence Erlbaum Associates, pp. 521-535.

[8] Fowler, C.A. (1979). "Perceptual Centers" in speech production and perception. Perception and Psychophysics, 25 (5), 375-388.

[9] Morton, J., Marcus, S. \& Frankish, C. (1976). Perceptual centers (P-Centers). Psychological Review, 83, 405-408.

[10]Tuller, B. \& Fowler, C.A. (1980). Some articulatory correlates of perceptual isochrony. Perception and Psychophysics, 27 (4), 277-283.

[11] Pompino-Marschall, B. (in press). The syllable as a prosodic unit and the so-called P-centre effect. To appear In G. Dogil (Ed.), Units and processes in speech perception. Berlin : Mouton de Gruyter. 